EESTI NSV TEADUSTE AKADEEMIA TOIMETISED. XI KOIDE FOOSIKALIS-MATEMAATILISTE JA TEHNILISTE TEADUSTE SEERIA. 1962, NR. 3

ИЗВЕСТИЯ АКАДЕМИИ НАУК ЭСТОНСКОИ ССР. ТОМ ХІ СЕРИЯ ФИЗИКО-МАТЕМАТИЧЕСКИХ И ТЕХНИЧЕСКИХ НАУК. 1962, № 3

\title{
К УТОЧНЕНИЮ РАСПРЕДЕЛЕНИЯ МАГНИТНОГО ПОЛЯ В ЭЛЕКТРИЧЕСКИХ МАШИНАХ С МАЛЫМ ВОЗДУШНЫМ ЗАЗОРОМ
}

\section{P. ПАРТС}

При анализе методом комформного преобразования влияния воздушного зазора на параметры электрической машины $[1,2,3,4]$ принимают внутреннюю поверхность статора или часть ее в качестве эквипотенциальной линии. Это было оправдано в той или иной мере у нормальных асинхронных машин. У асинхронных машин специального назначения, имеющих воздушный зазор значительно больше (во много раз - в 10 и больше раз) или меньше номинального воздушного зазора, внутренняя поверхность статора может и не быть эквипотенциальной поверхностью. Поэтому к формулам, полученным методом комформного преобразования, в подобных случаях нужно относиться критически. Так как в наиболее распространенных электрических машинах для автоматики, а также в индукционных регуляторах и других асинхронных двигателях встречается стремление инженеров-конструкторов к малым воздушным зазорам, то в настоящей статье целесообразно рассмотреть магнитное поле при наличии малого воздушного зазора. В самом общем внде данная задача не решается, поэтому принимаются следующие допущения: 1) электромагнитное поле, создаваемое только токами в статоре, является плоскопараллельным; 2) воздушный зазор имеет ничтожно малую величину, так что его магнитным сопротивленисм можно пренебречь; 3) проводник в пазу статора имеет бесконечно тонкую изоляцию от стенки паза; 4) проводник изготовлен из такого же ферромагнитного материала, из какого изготовлены пакеты статора и ротора, но, ради большей общности задачи, учитываем различную магнитную проницаемость в осевых направлениях; 5) по проводнику течет равномерно распределенный ток.

Для определения распределения магнитной индукции на полюсном делении будем исходить из магнитного поля одной секционной стороны однофазной обмотки с диаметральным шагом и числом пазов на полюс и фазу $q=1$ в пространстве, ограниченном плоскостями на расстоянии $\pm \frac{\tau}{2}$ и $\pm h$ от середины данной секционной стороны. Условие $\pm h$ указывает лишь на то, что магнитное поле вне машины отсутствует.

Из условия непрерывности магнитного поля получаем:

$$
\mu_{x} \frac{\partial H_{x}}{\partial x}+\mu_{y} \frac{\partial H_{y}}{\partial y}=0
$$


а из условия $\operatorname{rot} \overline{\dot{H}}=\overline{\dot{\delta}}$ получаем

$$
\frac{\partial H_{y}}{\partial x}-\frac{\partial H_{x}}{\partial y}=\delta_{z}
$$

где $\mu_{x}, \mu_{y}$ - составляющие магнитной проницаемости в осевых направлениях,

$x, y$ - текущие координаты,

$H_{x}, H_{y}$ - составляющие напряженности магнитного поля $H$ в осевых направлениях.

$\delta_{z}-$ аксиальная составляющая плотности тока.

После дифференцирования (1) по переменной $x$ и (2) по $y$ получаем после вычитания второго из первого дифференциальное уравнение для тангенциальной составляющей напряженности магнитного поля

$$
\frac{\mu_{x}}{\mu_{y}} \frac{\partial^{2} H_{x}}{\partial x^{2}}+\frac{\partial^{2} H_{x}}{\partial y^{2}}=-\frac{\partial \delta_{z}}{\partial y} .
$$

Пользуясь разложением плотности тока $\delta_{n}$ (в пазу) в двойной ряд Фурьє, получаем общее выражение для аксиальной составляющей плотности тока $\delta_{z}$ в вышеназванном пространстве

$$
\delta_{z}=\frac{8 \delta_{\mathrm{n}}}{h \tau} \sum_{m} \sum_{\nu} \frac{\sin \frac{m \pi}{2 h} b \sin v \frac{\pi}{\tau} a}{\frac{m \pi}{2 h} \cdot \frac{v \pi}{\tau}} \cos \frac{m \pi}{2 h} y \cos \frac{v \pi}{\tau} x,
$$

где $m$ - порядок тангенциальной пространственной гармонической,

$v$ - порядок радиальной (нормальной) пространственной гармонической,

$a, b, \tau, h$ - геометрические размеры, понятные из фигуры 1.

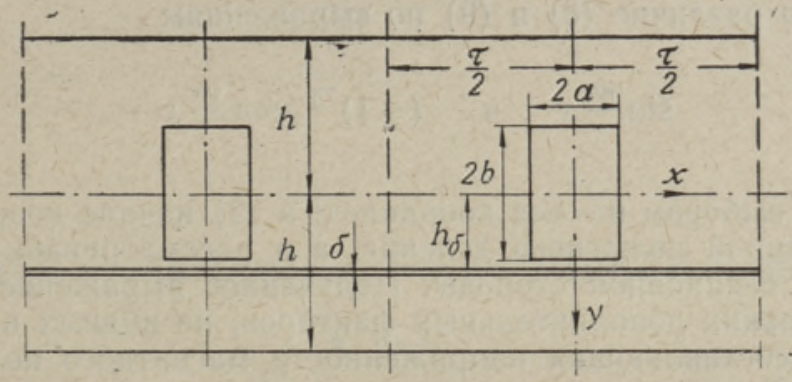

Фиг. 1.

С учетом (4) и (3) можно искать решение $H_{x}$ в следующем виде:

$$
H_{x}=-\sum_{m} \sum_{\nu} C_{m \nu} \sin \frac{m \pi}{2 h} y \cos \frac{v \pi}{\tau} x .
$$

При подстановке (5) и (4) в исходное уравнение (3) получаем после сокращений, что постоянная интегрирования 


$$
C_{m \nu}=\frac{8 \delta_{n}}{\pi h} \frac{\sin \frac{m \pi}{2 h} b \sin \frac{v \pi}{\tau} a}{\left[\left(\frac{m \pi}{2 h}\right)^{2}+\frac{\mu_{x}}{\mu_{y}}\left(\frac{v \pi}{\tau}\right)^{2}\right] \nu}
$$

Следовательно, тангенциальная составляющая напряженности магнитного поля

$$
H_{x}=-\frac{8 \delta_{n}}{\pi h} \sum_{m} \sum_{\nu} \frac{\sin \frac{m \pi}{2 h} b \sin \frac{v \pi}{\tau} a}{\left[\left(\frac{m \pi}{2 h}\right)^{2}+\frac{\mu_{x}}{\mu_{y}}\left(\frac{\nu \pi}{\tau}\right)^{2}\right]_{\nu}} \sin \frac{m \pi}{2 h} y \cos \frac{v \pi}{\tau} x .
$$

Пользуясь выражениями (1) и (7), получаем и выражение для радиальной составляющей напряженности магнитного поля

$$
H_{y}=\frac{16 \delta_{n}}{\pi \tau} \frac{\mu_{x}}{\mu_{y}} \sum_{m} \sum_{\nu} \frac{\sin \frac{m \pi}{2 h} b \sin \frac{\nu \pi}{\tau} a}{\left[\left(\frac{m \pi}{2 h}\right)^{2}+\frac{\mu_{x}}{\mu_{y}}\left(\frac{\nu \pi}{\tau}\right)^{2}\right] m} \cos \frac{m \pi}{2 h} y \sin \frac{\nu \pi}{\tau} x .
$$

Ниже для сравнения приведено выражение радиальной составляющей напряженности магнитного поля, из которого мы исходим ежедневно при расчете нормальных асинхронных машин $\left[{ }^{4}\right.$, стр. 290]

$$
H_{y}=\frac{f_{\mathrm{k}}}{\delta}=\frac{\frac{4}{\pi} \frac{\sqrt{2}}{2} I_{s} \cdot s_{\mathrm{n}}}{\delta} \sum_{\nu}(-1)^{\frac{\nu-1}{2}} \cos \frac{v \pi}{\tau} x,
$$

где $\delta$ - длина воздушного зазора (только в случае выражения (9)), $f_{k}$ - н. с. на полюсном делении,

$I_{s}-$ ток в витке,

$s_{\mathrm{n}}-$ число витков в пазу.

Заметим, что различие (8) и (9) по выражениям

$$
\sin \frac{v \pi}{\tau} x \quad \text { и } \quad(-1)^{\frac{\nu-1}{2}} \cos \frac{v \pi}{\tau} x
$$

объясняется выбором начала координат: в (9) начало координат совпадает с серединой полюсного деления, а в рассматриваемом случае c серединой секционной стороны. Полученное выражение (8) показывает, от скольких дополнительных факторов, не видных в (9), зависит радиальная составляющая напряженности магнитного поля в воздушном зазоре. Разумеется, что от этих же факторов зависят амплитуды магнитной индукции основной гармонической и высших пространственных гармонических. 


\title{
Выводы
}

1. Радиальная (тангенциальная) составляющая напряженности магнитного поля в воздушном зазоре зависит не только от длины воздушного зазора и от полюсного деления, но и от магнитных свойств материала ротора и статора, от ширины и высоты паза, от радиальной толщины статора и ротора. Тем самым показывается и ограниченность метода комформных преобразований.

2. Решение даст возможность учесть состав высших пространственных гармонических, обусловливающих дополнительные потери и искажающих кривую Э.Д.С.

3. Полученное решение не связано с частными размерами машины, поэтому данное решение определяет магнитное поле единичного прямоугольного провода из немагнитного материала в воздухе, если магнитную проницаемость стали заменить магнитной проницаемостью воздуха. Методом наложения данное решение может быть распространено и на систему прямоугольных проводов в воздухе.

\section{ЛИТЕР А Т Р Р А}

1. И. М. А снин, Расчеты электромагнитных полей, Изд. ВЭТА, Л., 1939.

2. J. Gib b s. Induction and synchronous motors with unlaminated rotors, The Journal of IEE, 1948, part II, vol. 95, No. 46, p. 411.

3. H. W. T a y lor, P. T. C o e, Some problems of calculation in electrical machine design involving elliptic function, Philosophical Magazine and Journal of Science, 1928, vol. 6 , No. 34, p. 100.

4. Л. М. Пиотровски й, Электрические машины, Госэнергоиздат, Л., 1949.
Ннститут энергетики
Академии наук Эстонской ССР
Поступила в редакцию
1. II 1961

\section{MAGNETVĂLJA JAGUNEMINE OLIVÄIKESE ÖHUPILUGA ELEKTRIMASINATES}

\author{
R. Parts \\ Resümee
}

Artiklis tutvustatakse vähe esineva kahekordse Fourier' rea kasutamist magnetvälja jagunemise määramiseks sellises keskkonnas, mille magnetiline läbitavus telgede suunas vôib olla erinev. Toodud lahendi üldine kuju võimaldab magnetvälja jagunemise probleemi lahendada ka õhkkeskkonnas, millesse on paigutatud rööpkülikukujuliste ristlőigetega mittemagnetilisest materjalist juhtmed, kuna iga juhtme tekitatud magnetvälja võib lahendada samas keskkonnas teistest juhtmetest sōltumatult. Antud lahendis on hästi näha, kuidas üliväikese ōhupiluga elektrimasinates üksikud masina geomeetrilised mõõtmed (uurde laius ja kõrgus, pooluse jaotus ning magnetahela laius 2h) mõjustavad kõrgemate ruumiliste harmooniliste koosseisude tekkimist ning viimaste amplituudi suurust. 


\section{DISTRIBUTION OF MAGNETIC FIELD IN ELECTRIC MACHINES WITH PARTICULARLY SMALL SLOT}

\section{R. Parts}

\section{Summary}

The author presents a method of application of the rarely-occurring Fourier doubleseries for determining the distribution of the magnetic field in such medium where the magnetic permeability in direction to the axis may be different. The general form of the solution presented may also be applied at the determination of the distribution of the magnetic field in air, with conductors which are of non-magnetic material and parallelogrammic in cross-section, as the magnetic field formed by each conductor can be determined in the same medium independently of the other conductors. The presented solution shows how the separate geometrical dimensions of an electric machine with an extremely small slot (width and height of slot, distribution of pole and width of magnetic chain $2 \mathrm{~h}$ ) influence the formation of higher three-dimensional harmonics and the value of their amplitude.

\section{Academy of Sciences of the Estonian S.S.R.,} Institute of Energetics

Received

Feb. 1st, 1961 